\title{
Genome sequencing reveals how Shigella sonnei spread around the world
}

$\mathrm{n}$ international research team has
demonstrated that the current
population of Shigella sonnei diversified in Europe from a common ancestor and that different lineages then became established on other continents, in a new paper published in Nature Genetics.

S. sonnei is one of the primary causes of dysentery in developed countries, and is now replacing $S$. flexneri as the leading cause of the illness in developing countries. Treating dysentery caused by $S$. sonnei is becoming increasingly difficult as multidrug-resistant strains become established. Understanding the epidemiology and pathogenicity of these bacterial populations is therefore important.

Kathryn Holt (University of Melbourne, Australia) and colleagues sequenced the genomes of 132 samples of S. sonnei that were isolated between 1948 and 2008 from Europe, Asia, Africa, Central America and South America. Four distinct lineages of S. sonnei were identified. Although the lineages are characterized by hundreds of different single nucleotide polymorphisms, the gene content only had minor differences.

The most recent common ancestor for the different lineages existed in Europe less than 500 years ago-between 1554 and 1763. Lineages I and II had emerged by the early $19^{\text {th }}$ century, with lineages III and IV appearing in the late $19^{\text {th }}$ century. The analysis revealed strong regional clustering of the different lineages. Europe had the richest diversity, with all four lineages being found here, which indicates that S. sonnei diversified in Europe. Lineage III is the dominant form in Asia, Africa, Central America and South America, followed by lineage II, with a few examples of lineage I found in Africa. One isolate of lineage IV was found in France.

"The phylogenetic analysis indicates that all contemporary S. sonnei infections are caused by a small number of clones that have recently become globally

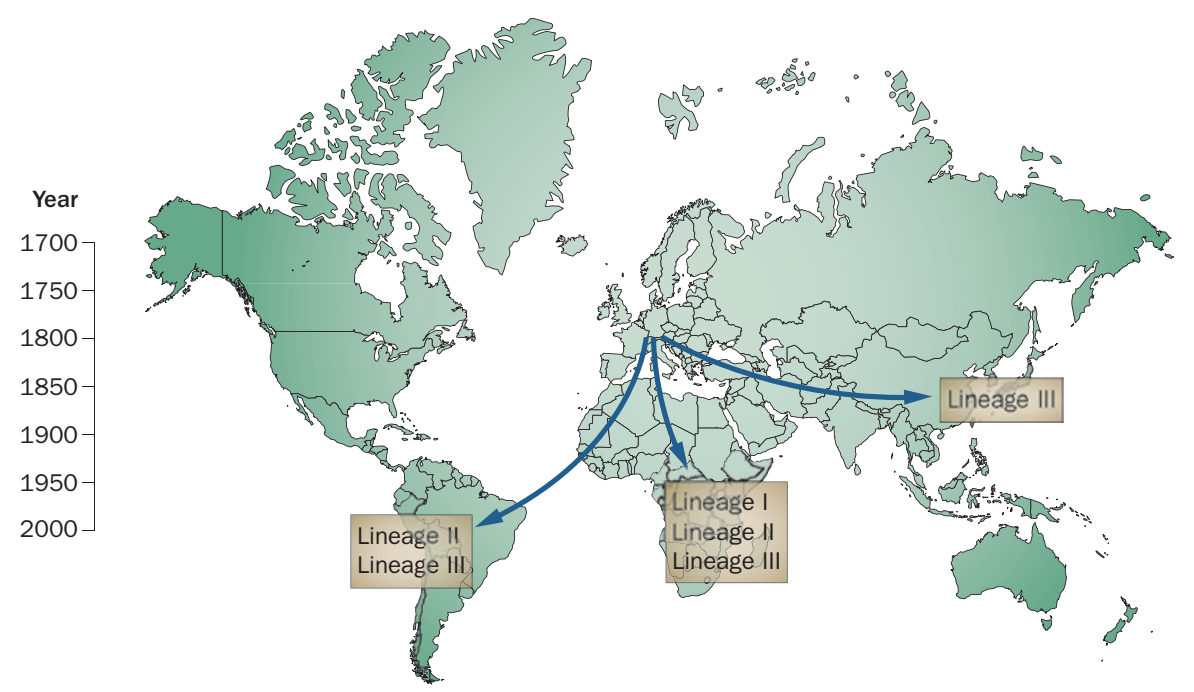

Genetic analysis has revealed that the different Shigella sonnei lineages diversified in Europe and then spread to other continents.

dispersed," write the authors. This global dissemination was seemingly driven by the distribution of antimicrobial resistance genes and mutations. The researchers showed that the establishment of lineage III (the most prevalent lineage) outside of Europe was associated with the presence of transposon Tn7 and class II integrons that encode resistance to multiple antimicrobials. "The work raises the issue of the impact of antibiotics in driving the evolution and success of a pathogen," notes Philippe Sansonetti, a leading expert in Shigella who is based at the Institut Pasteur, Paris, France. Antimicrobials are the current recommended treatment for dysentery, but because of high levels of drug resistance, this strategy might not be effective in cases of dysentery caused by S. sonnei.

In addition, the authors note that exposure to water contaminated with Plesiomanos shigelloides has been proposed to protect humans from S. sonnei infection. This phenomenon is probably the result of $P$. shigelloides and $S$. sonnei having very similar $\mathrm{O}$ antigens, which can cross-react to result in cross-protection. As countries develop economically and water quality improves, humans are less likely to be exposed to P. shigelloides. Passive cross-protection by environmental immunization with P. shigelloides therefore declines, which might explain the increase in S. sonnei infections in developing countries.

"The study is important in a period of rediscussion of the vaccine priorities in the field of prophylaxis against enteric pathogens," says Sansonetti. Indeed, the authors state that a vaccine is becoming increasingly important for the control and long-term prevention of dysentery caused by $S$. sonnei as the incidence of this disease and drug resistance increases. A vaccine against $S$. sonnei infection should be possible, as all the lineages have the same $\mathrm{O}$ antigen that has been shown to be a successful target for vaccines. "Vaccination and improved hygiene standards will be pivotal in eliminating $S$. sonnei infections in industrializing countries," conclude Holt and co-workers.

Claire Greenhill 